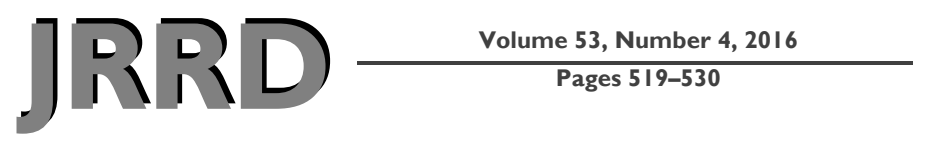

\title{
Case study: Head orientation and neck electromyography for cursor control in persons with high cervical tetraplegia
}

\author{
Matthew R. Williams, PhD; ${ }^{*}$ Robert F. Kirsch, PhD $^{1-2}$ \\ ${ }^{1}$ Department of Biomedical Engineering, Case Western Reserve University, Cleveland, OH; Cleveland FES Center, \\ Cleveland, OH; and Louis Stokes Cleveland Department of Veterans Affairs Medical Center, Cleveland, OH; \\ ${ }^{2}$ MetroHealth Medical Center, Cleveland, $\mathrm{OH}$
}

\begin{abstract}
We evaluated the ability of an individual with a high cervical spinal cord injury (SCI) to control a cursor on a computer screen using two different user interfaces: (1) head movements measured via a head-mounted orientation sensor and (2) electromyography (EMG) signals from four head and neck muscles acquired using a 4-channel implanted upper-limb neuroprosthesis that had been deployed in an earlier study. The subject moved the cursor to a set of targets on the screen in a two-dimensional, center-out, target-acquisition task, and his performance was evaluated with a variety of performance measures to assess both position and velocity control accuracy. The subject's performance with both command sources was also compared with the performance of a group of nondisabled subjects. Head orientation provided more accurate performance but was less responsive than EMG. Both command sources showed some directionally dependent performance, with movement to diagonally located targets being performed by a series of sequential motions rather than via straight paths. Overall, the SCI subject's performance with each command source was similar to that reported for a nondisabled population using the same interfaces and performing the same task.
\end{abstract}

Key words: command source, cursor control, electromyography, Fitts' Law, head orientation, human-computer interface, interface evaluation, performance measure, spinal cord injury, tetraplegia.

\section{INTRODUCTION}

A significant loss of function can occur as a result of a high cervical spinal cord injury (SCI), affecting both the injured persons and their caregivers and families. Approximately 11,000 new SCIs occur each year, and currently about 250,000 individuals with SCI live in the United States [1]. Approximately 18 percent of these cases are classified as high tetraplegia, defined as SCI at cervical levels 1 to 4 , resulting in significant paralysis from the shoulders downward [1]. Despite the injury and the resulting voluntary movement limitations, several means of operating user interfaces for the command and control of assistive technology remain available to individuals with high SCI. The aim of this article is to study the performance of two interfaces to control computer cursor position that would be immediately deployable in this population.

\footnotetext{
Abbreviations: $\mathrm{EMG}=$ electromyography, $\mathrm{EMG}_{\mathrm{mvc}}=$ maximum voluntary contraction EMG, ID = Index of Difficulty, MT = movement time, $\mathrm{SCI}=$ spinal cord injury.

*Address all correspondence to Matthew R. Williams, PhD; Louis Stokes Cleveland VA Medical Center, 10701 East Blvd, Cleveland, OH 44106; 216-392-5062; fax: 216231-3258. Email: mwilliams@fescenter.org

http://dx.doi.org/10.1682/JRRD.2014.10.0244
} 
Immense potential benefits could be had by restoring computer operation to individuals with a high-level SCI. Roughly half (55.5\%) of all jobs required computer use as of 2003 [2], and in 2015, it was shown that 78 percent of the rapidly growing middle-skills job sector requires computer operation [3]. Despite these potential opportunities, only 24.2 percent of individuals with tetraplegia are employed a decade after their injury [1]. Beyond employment, given the ubiquitous nature of the Internet in everyday life, the need for computer operation for communication, education, commerce, and leisure is critical [4]. Not surprisingly, people with an SCI have reported significantly higher quality of life measures when they have Internet access, and of those with Internet access, approximately 81 percent use the Internet weekly or more often [5].

Several computer interfaces for cursor control that are useable by the SCI community have been developed over the years, though limited quantitative analysis of these systems with this population has been performed. The possible voluntary actions that have been employed as computer interfaces for subjects with SCI include mechanical controls, such as chin-operated devices [6]; tongue-controlled systems and mouthsticks [7-8]; headposition controls [9-10]; and voice commands [11]. Some studies have looked into electromyography (EMG), both to determine head angles using neck EMG signals [12-13] and as an interface for controlling computer cursor position [14-16]. Little quantitative assessment was performed in these early studies; they were often inconsistent with current human-computer interface assessment literature, primarily intended to measure text-entry performance, or not applicable to individuals with severe paralysis such as seen in high tetraplegia.

Fitts first quantified human target-acquisition performance in a 1954 seminal article describing the bounds of upper-limb accuracy and speed in a single degree-offreedom tapping task [17]. This idea was further expanded to the realm of human-computer interface analysis in 1978 by Card et al., who showed that humancommanded cursor motion, like hand motion, follows a Fitts' Law model [18]. Since then, several common computer interfaces have been similarly studied, including mice [18-19], joysticks [18], and trackballs [19]. However, few computer interfaces applicable to individuals with SCI have been similarly quantified. Headcommanded cursor position control has been investigated by Jagacinski and Monk [20], Radwin et al. [21], and LoPresti et al. [22-23], with performance assessed using a two-dimensional target-acquisition task similar to the one used in this study. Other than these, rigorous quantitative analysis of computer interfaces specifically applicable to individuals with SCI has not been performed.

This case study quantitatively assesses the performance of two human-computer user interfaces by one individual with high tetraplegia: (1) head orientation and (2) EMG from four muscles of the face and neck acquired from an implanted EMG system. The performances of these two command sources were also compared to previously published performance data from nondisabled subjects conducting the same task with the same user interfaces [24].

\section{METHODS}

The subject examined in this study was a participant in a larger study to develop a neuroprosthesis for restoration of hand and arm function following high cervical SCI and as a result had an implanted device to both record EMG and stimulate the muscles of the arm [25]. The subject had a cervical 1 to 2 level injury with BrownSequard syndrome, resulting in complete motor paralysis in the right upper limb. The subject was more than $10 \mathrm{yr}$ postinjury and was otherwise healthy and able in regards to his ability to voluntarily control neck and facial muscles and move his head to desired head orientation angles. The subject used his implanted system daily, approximately 1-2 h per day, to operate a computer cursor via EMG, primarily for entertainment purposes. Pertinent human subject protections were observed, including obtaining informed consent and approval by the Metro Health Medical Center Institutional Review Board.

The subject participated in a two-dimensional targetacquisition experiment similar to that used by Fitts and others that entailed moving a computer cursor (arrow) to circular targets randomly presented about the center of the screen (Figures 1 and 2(a)) using either head orientation or EMG as command inputs. Six performance measures were used to quantify command source effectiveness: throughput, path efficiency, overshoot, reaction time, average speed, and direction ratio.

\section{Task}

Similar to previous studies [20-21,23-24], a centerout task was used, with targets radially distributed across a 1,000 by 1,000 pixel field in eight directions. The task 


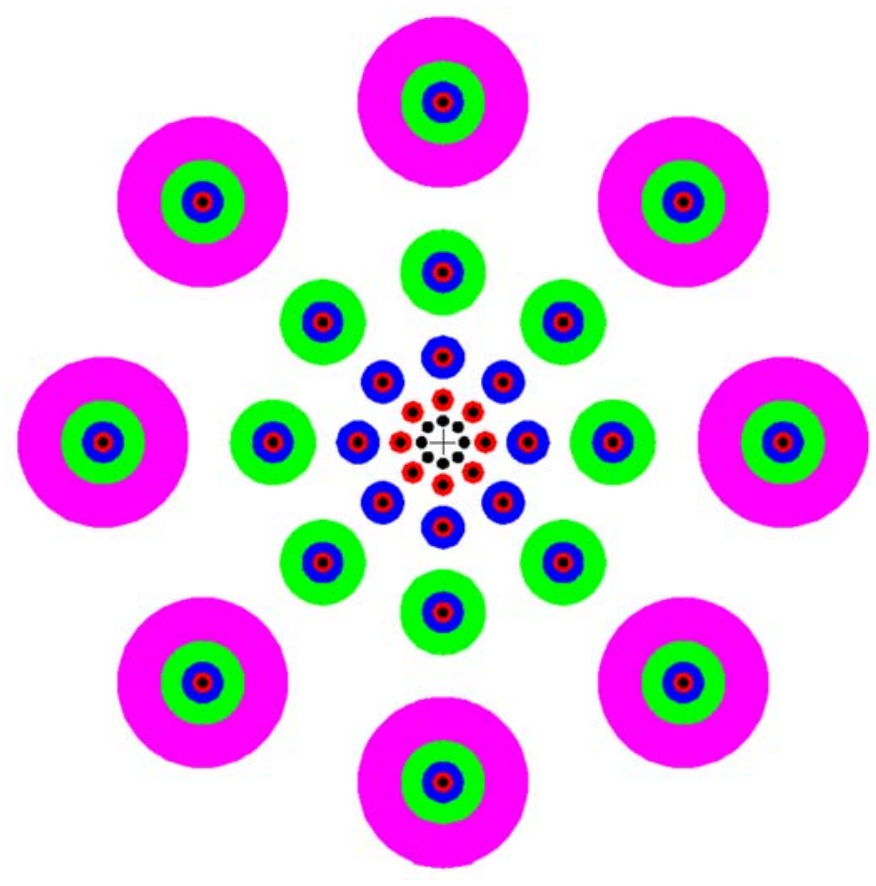

Figure 1.

All 120 targets presented to participant. Targets were presented to subjects as green on black background (as shown in Figure 2(a)) but are shown here in different colors to better illustrate relative sizes of overlapping targets.

used 120 total targets consisting of five fixed distances from the central starting point and five fixed target diameters (Table 1). Targets were evenly spaced about the central starting point on one of eight radial lines. Targets were presented randomly, varying for each trial in both size and location about the center of the screen. Figure 1 illustrates the sizes and target locations used in the study.

To indicate controlled placement of the cursor within the target, the subject had to keep the cursor within the target region for $2 \mathrm{~s}$ to complete the task. This dwell time in the target was not factored into the overall movement time used to assess performance. After successful target acquisition, the trial was reset, the cursor automatically re-centered, and the target removed from the screen before presentation of a subsequent target. This task required user control over a large range, in different directions, and with different degrees of accuracy (i.e., target sizes). Both command sources were evaluated over 4 blocks of 120 targets each. A 5 min break between blocks was used to reduce fatigue and help the subject maintain focus on the task.

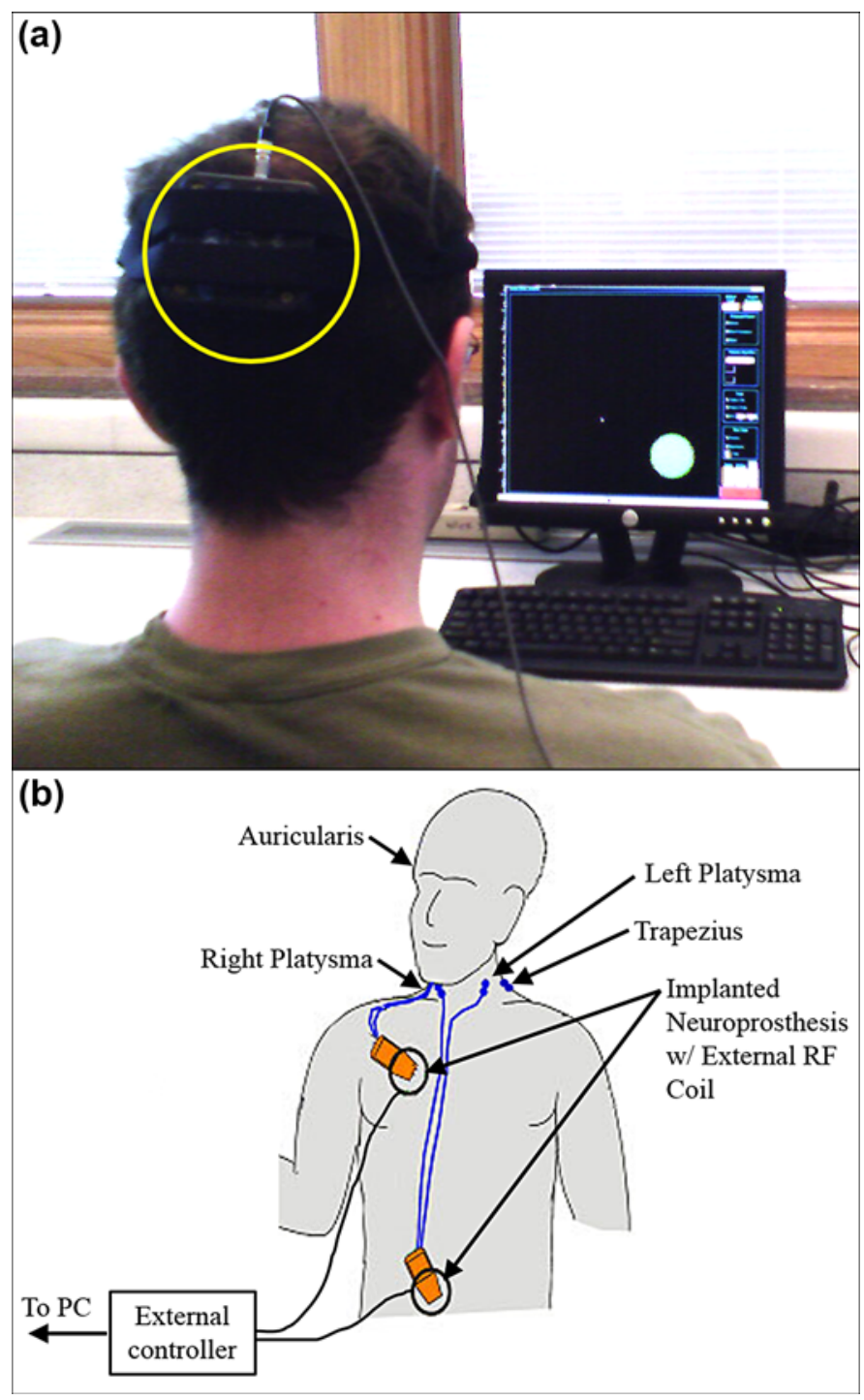

Figure 2.

(a) Head orientation sensor on subject's head (circled) while performing target acquisition task. (b) Diagram of implanted electromyography (EMG) recording system and muscles used for EMG cursor control. $\mathrm{RF}=$ radio frequency, $\mathrm{PC}=$ personal computer.

Table 1.

Target widths and distances (in pixels) and ensuing Indices of Difficulty.

\begin{tabular}{cccccc}
\hline $\begin{array}{c}\text { Distance } \\
\text { (pixels) }\end{array}$ & \multicolumn{6}{c}{ Width (pixels) } \\
\cline { 2 - 6 } & $\mathbf{1 3}$ & $\mathbf{2 5}$ & $\mathbf{5 0}$ & $\mathbf{1 0 0}$ & $\mathbf{2 0 0}$ \\
\hline 25 & 1.58 & - & - & - & - \\
50 & 2.32 & 1.58 & - & - & - \\
100 & 3.17 & 2.32 & 1.58 & - & - \\
200 & 4.09 & 3.17 & 2.32 & 1.58 & - \\
400 & 5.04 & 4.09 & 3.17 & 2.32 & 1.58 \\
\hline \hline
\end{tabular}


Circular targets were used to maintain a constant target width regardless of approach angle [26]. Consistent with similar studies, the Shannon form of the Index of Difficulty (ID) (Equation) was used to evaluate performance across the different combinations of target width and distance $[24,26]$ :

$$
\mathrm{ID}=\log _{2}\left(\frac{\mathrm{D}}{\mathrm{W}}+1\right)
$$

The 15 IDs from five different target distances (D) and five target diameters (W) are shown in Table 1 and span from 1.58 to 5.0 pixels. The target distance was measured as the straight-line distance between where the cursor entered the target and the central starting location. The subject was instructed to move the cursor from the central starting position to the target as quickly and accurately as possible. The subject was otherwise not specifically instructed about the cursor speed or path.

\section{Command Sources}

The command sources selected for this study, head orientation and neck and face muscle EMG signals, were chosen for testing as user interfaces because they are able to be voluntarily controlled after a high cervical SCI and the movements associated with these actions are generally not directly employed to perform activities of daily living, such as eating, communicating, or grooming [27], minimizing interference with these tasks.

As in previous studies [9,23-24], a three-axis orientation sensor (MicroStrain 3DM; Williston, Vermont) worn on the back of the head was used to measure head orientation (Figure 2(a)). This small, lightweight sensor ( 75 g; $89 \times 6 \times 25 \mathrm{~mm}$ ) did not interfere with the subject's head movements. Vertical cursor movement was controlled using head pitch (nodding), and horizontal movement was commanded by head yaw (looking left/ right). Head roll was ignored in the study.

EMG signals were recorded by a neuroprosthesis implanted for restoration of hand and arm function in an earlier study [28]. EMG signals from a total of four muscles were used in the command interface because this was the number of channels provided by the implanted neuroprosthesis. Signals were recorded from the right and left platysma muscles (for right and left motion commands), the right auricularis (downward movement commands), and the left trapezius (for upward commands)
(Figure 2(b)). These particular muscles were not used to measure head orientation similar to previous studies [1213], but as independent voluntary signals. Differential, bipolar implanted EMG signals were amplified by a gain of 2,000, bandpass filtered over 70 to $1,000 \mathrm{~Hz}$, converted via 12-bit analog-to-digital conversion, and bin rectified over a $10 \mathrm{~ms}$ window by the implanted neuroprosthesis. This processed signal was then telemetered at $12.5 \mathrm{~Hz}$ to an external implant controller via radio frequency coils located on the surface of the torso. The signal was then sent to the computer presenting the experimental task via the serial port (10 ms packet interval). Using a $60 \mathrm{~ms}$ loop time, the complete system (including signal acquisition, processing, and transmission) operated in real-time, with no lag in cursor motion noticeable by the subject.

\section{Velocity Algorithm}

A proportional velocity gated-ramp algorithm was used to convert user signals (head orientation or EMG) into cursor velocity commands. This algorithm has been shown in previous studies to perform better than direct position control because it requires less fine motor accuracy and is less fatiguing [25]. User signals above a set detection threshold were converted into a parabolic cursor velocity command such that cursor speed was proportional to the square of the ratio of the signal amplitude to the overall dynamic range of the signal. This allowed for both coarse, rapid motion to distant targets as well as fine, slow movements at low command levels. Maximum cursor speed was fixed at 500 pixels per second (pps). The total range for head orientation was a cone $60^{\circ}$ wide, centered on a neutral orientation while the subject faced the center of the computer screen. Maximum EMG amplitude was set at 70 percent of the maximum voluntary contraction EMG (EMG $\left.\mathrm{Evc}_{\mathrm{mv}}\right)$. This yielded a level of effort that was sustainable over the run of the experiment without producing fatigue.

A threshold of $10^{\circ}$ off-center was used for head orientation to allow for the few degrees of natural drift that has been observed while individuals attempt to hold their head motionless [20]. The threshold for EMG command onset was fixed at 20 percent of the $E M G_{m v c}$ and included a shifting baseline calculation to account for long-period changes in EMG magnitudes over the course of the experiment. Complete details regarding the velocity algorithm can be found in Williams and Kirsch [24]. Due to crosstalk observed between the left platysma and 
trapezius electrodes, the velocity algorithm for leftward and upward motion using EMG signals was adjusted such that only the greater of these signals would produce motion in their respective direction (i.e., a winner-takeall approach).

\section{Performance Metrics}

To more fully assess command source operation, we used several previously developed performance measures. These include measures of overall performance as well those used to evaluate more detailed aspects of user interface performance $[19,24]$. While the complete details of these performance measures can be found in Williams and Kirsch [24], briefly, they consist of the throughput (an overall summary measure of information transfer rate), path efficiency (a measure of the path straightness to the target), overshoot (the number of times the subject passes through the target without stopping), reaction time (the time from the start of the trial to the beginning of cursor movement), and average speed (the nonzero speed of the cursor averaged over the trial). For each of these measures, a direction ratio was also calculated to illustrate the effect on the performance of each command source of moving in two dimensions (diagonal directions), which requires two separate simultaneous commands, versus moving in one dimension (horizontally or vertically), which requires a single command.

\section{Protocol}

Subject performance with each of the two command sources was assessed in separate experimental sessions on different days. Each experiment started with 5 min of unguided "free movement" practice with the command source to be tested, with 4 blocks of 120 trials each following. The first block was considered as recorded practice, and the results were not used to evaluate performance. Subject performance was quantified using the data from blocks 2-4. Preliminary testing with nondisabled subjects had indicated that while performance increased between blocks 1 and 2 for some subjects $(p<0.05)$, performance from block 2 onward plateaued, with no statistical performance improvement observed across the last three blocks ( $p=0.62$ ). These blocks, being similar in performance, were lumped for the analyses. The experiment lasted approximately $2 \mathrm{~h}$, including setup, practice, the experiment itself, and rest periods in between each command source.
Performance measures for each of the eight target directions (directionally dependent performance) were tested for normality and were found to fit a normal distribution. Performance for each command source was compared with the other (within-subject comparison) using paired single-tailed $t$-tests with a 95 percent confidence level. The SCI subject's individual performance with both command sources was also compared with previously published nondisabled performance [24] (betweensubject populations comparison) using the same test and confidence.

\section{RESULTS}

Figure 3 illustrates typical two-dimensional cursor trajectories (Figure 3 (a)-(b)), the position of the cursor over the trial (Figure $\mathbf{3}$ (c)-(d)), and the user input signals over the trial (Figure 3(e)-(f)). Each column represents a command source (head orientation to the left and EMG to the right), and the representative trials show motion to the same target (location, distance, and diameter). The head orientation-commanded motion (left column) was characterized by sequential commands in yaw $(y)$ first and then pitch $(x)$ (Figure 3(e)), leading to time-offset $x$ and $y$ commands (Figure 3(c)) that produced clearly separate $x$ and $y$ components in the two-dimensional cursor trajectory (Figure 3(a)). The EMG user command signals (Figure 3 , right column) produced even more obviously separate $x$ and $y$ commands. The various EMG signals (Figure 3(f)) were consistently activated at different times, resulting in significantly time-offset $x$ and later $y$ cursor movements (Figure 3 (d)) that produced distinctly directional twodimensional cursor movements (Figure 3(b)).

Figure 4 shows the velocity histograms and cursor motions for all 120 targets. Figure 4(a)-(b) indicate head orientation-commanded cursor movements, while Figure 4(c)-(d) indicate EMG-commanded cursor movements. Figure 4(a) and (c) show the cursor movements and Figure 4(b) and (e) show the corresponding velocity histograms. Head orientation-commanded motions (Figure 4(a)) exhibited very straight movements to targets that required only pure $x$ or $y$ cursor motions (e.g., up, down, left, and right). Motions toward the targets at $45^{\circ}$ from one of the major axes (those requiring simultaneous control of head orientation in two dimensions) were achieved in some cases by relatively diagonal motions and in some cases by separate movement components along the $x$ and $y$ directions. On the other hand, the EMG-commanded 

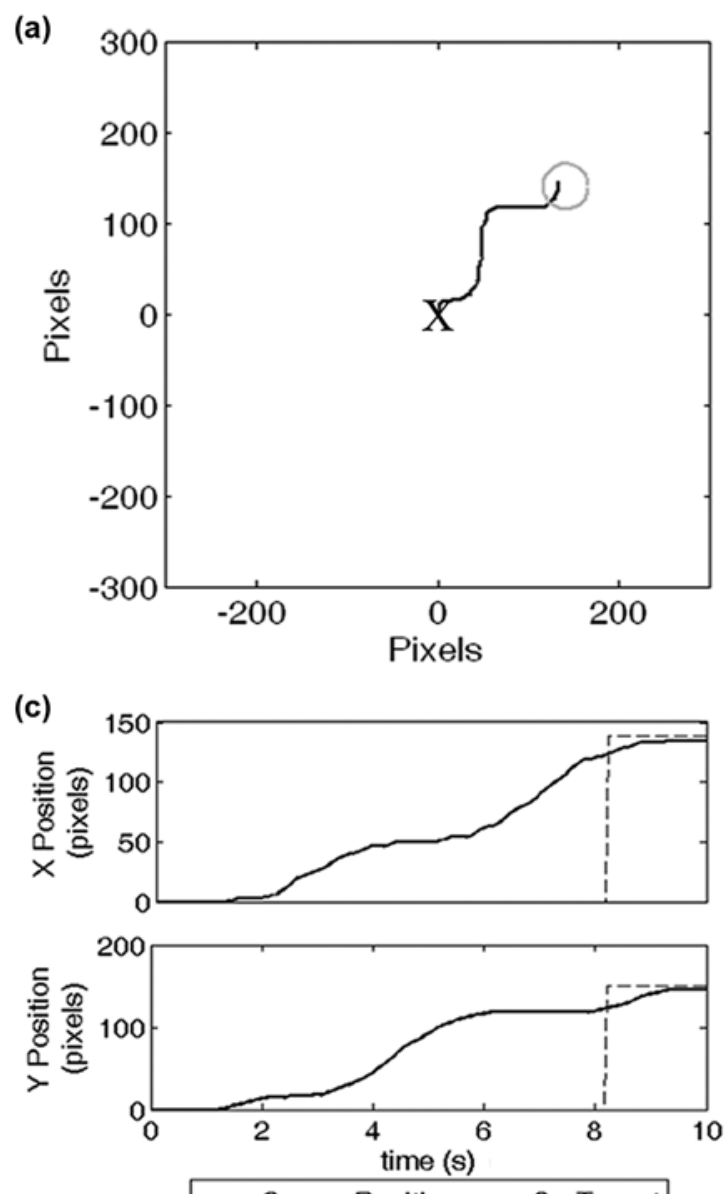

- Cursor Position ---On Target

(e)

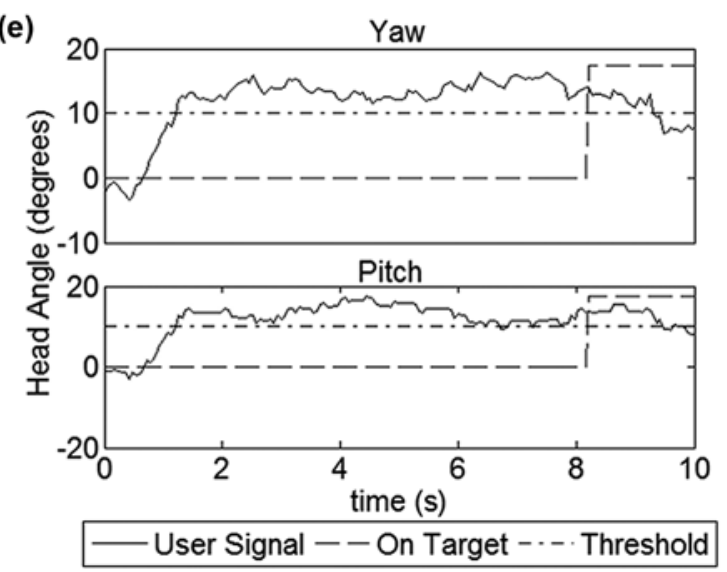

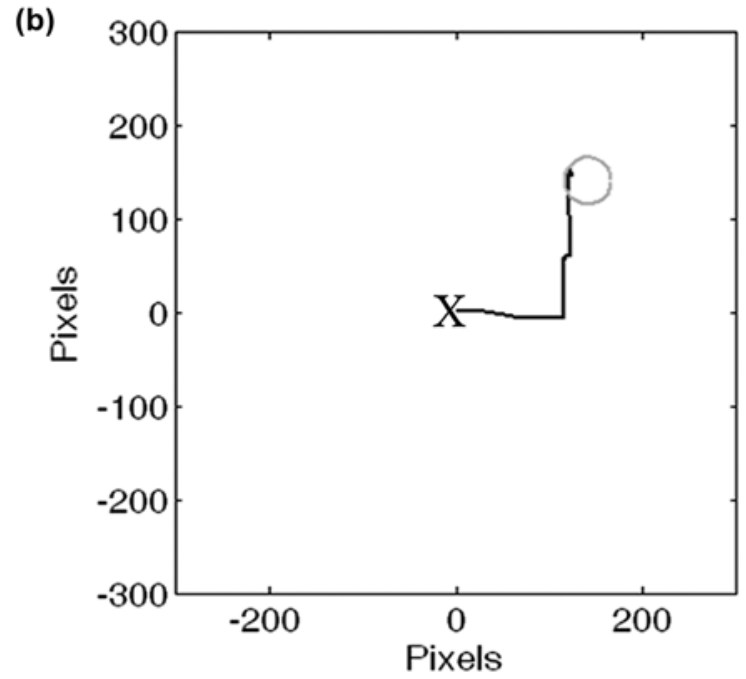
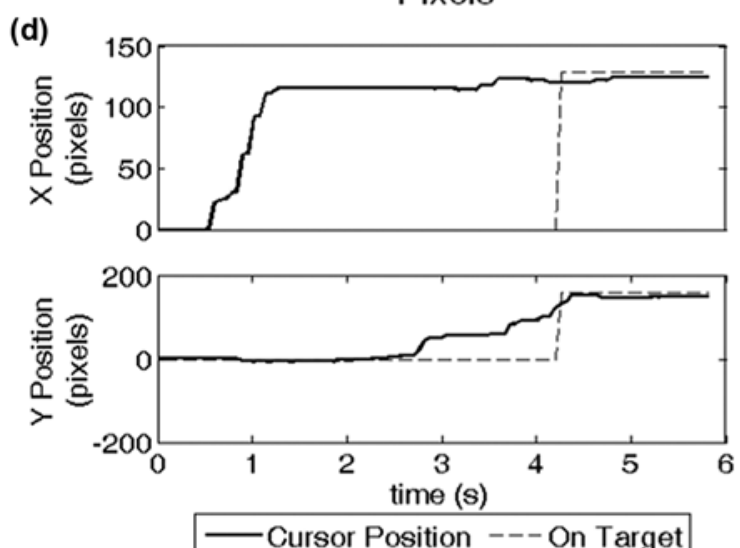

(f)

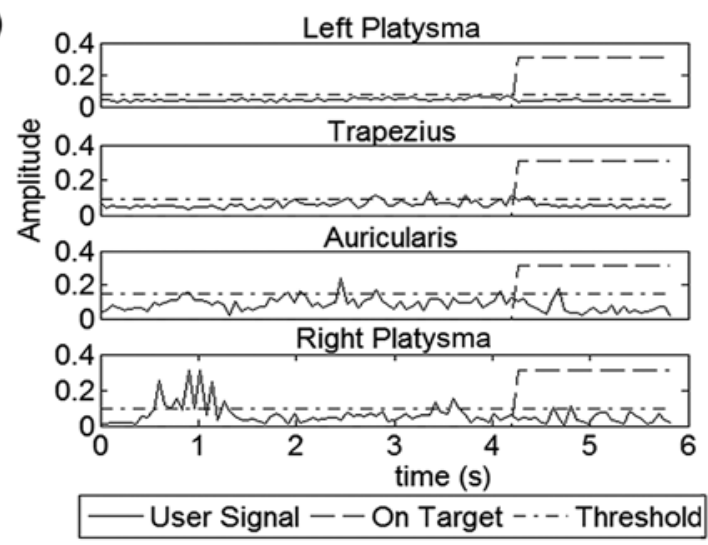

Figure 3.

Example of participant with spinal cord injury's cursor movements to identical target using (a), (c), (e) head orientation and (b), (d), (f) electromyography (EMG) as command interface. (a)-(b) is cursor path to target, starting from " $x$ " at center of circular target area. (c)-(d) illustrates $x$ and $y$ positions of cursor over trial. (e)-(f) shows command signals generated by user, with command thresholds indicated by dash-dot line. Rise in dashed line in (c)-(f) indicates when cursor is within target region. Of particular note is more constant user signal while using head orientation as command source compared with sequence of more intermittent commands while using EMG. 
(a)

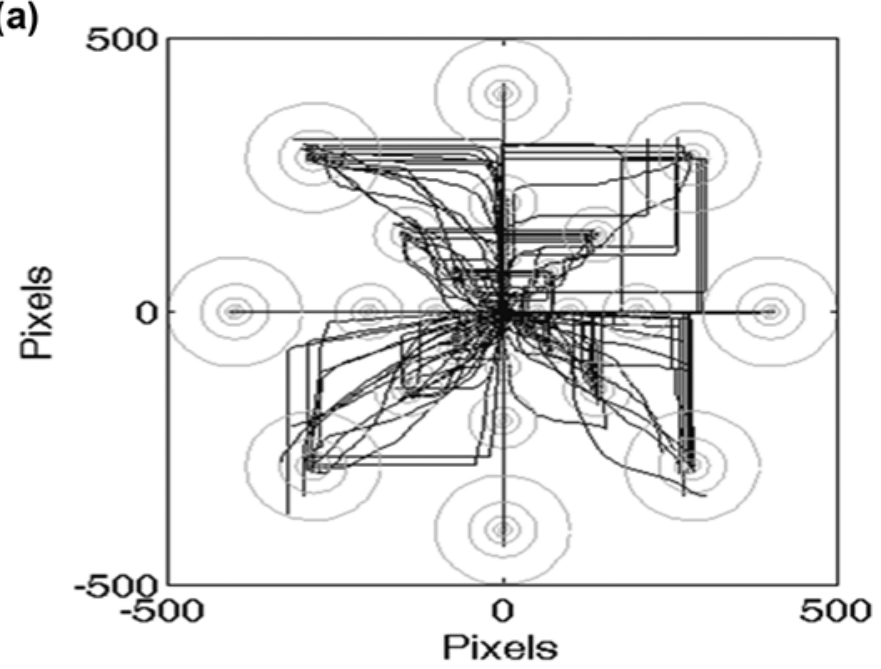

(c)

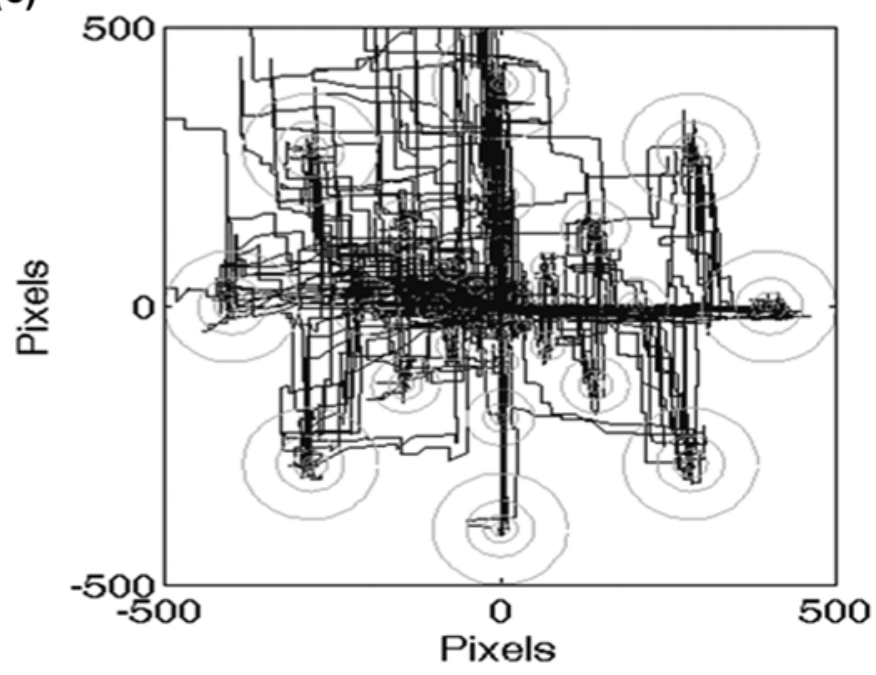

(b)

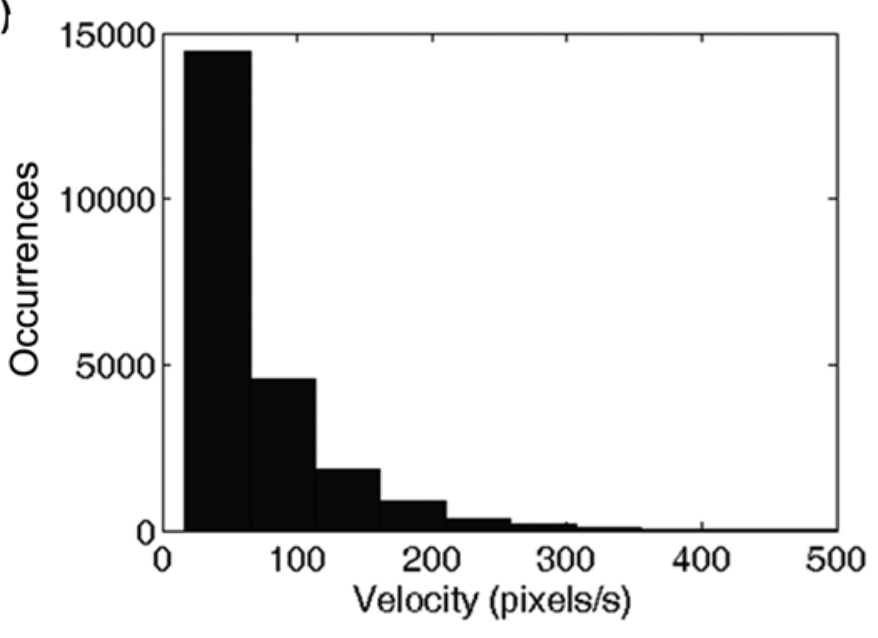

(d)

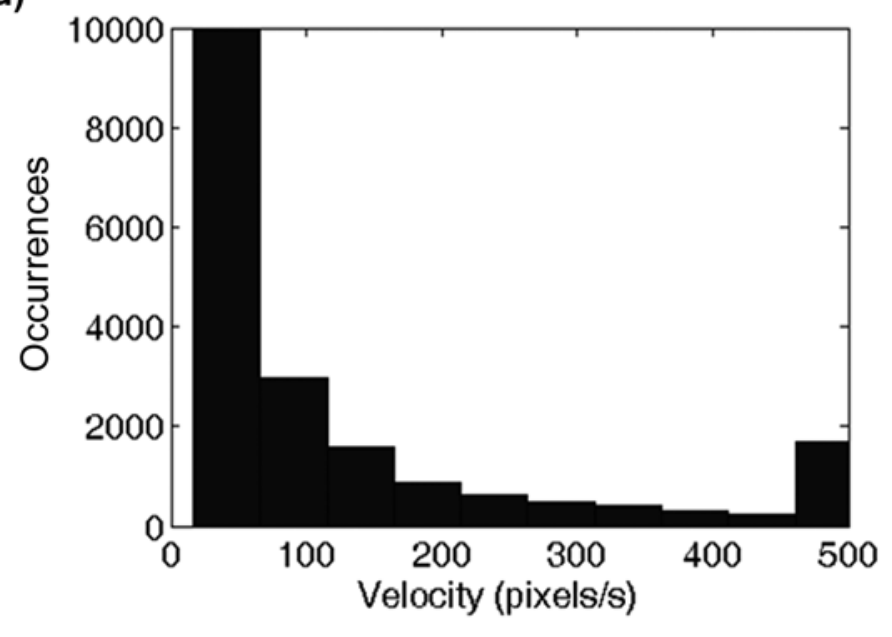

Figure 4.

Cursor traces and velocity histograms (left and right columns, respectively) for all experimental blocks. (a)-(b) Head orientation. (c)-(d) Electromyography.

cursor movements (Figure 4(c)) were almost always achieved by sequential movements, moving first in one direction and then the other, producing pronounced "boxy" trajectories. The effect of the crosstalk between the left platysma and trapezius EMG signals (controlling left and upward motions, respectively) can also be seen in the more erratic traces in the upper-left portion of the figure. The corresponding velocity histograms are shown in Figure 4(b) for head orientation and Figure 4(d) for EMG signals. Similar speed characteristics were seen at slower speeds for both interfaces, but more occurrences of the highest-permissible speed (500 pps) were seen for EMG, which is indicative of speed limiting for use of this command source.

Figure 5 shows the relationship between movement time (MT) and ID. For both command sources, MT increased roughly in proportion to ID. The correlations between MT and ID were not significantly different for head orientation $\left(R^{2}=0.68\right)$ or EMG-commanded $\left(R^{2}=\right.$ $0.71)$ movements $(p=0.49)$ (Figure 5(a)-(b)). The correlations of MT and ID for both command sources were found to significantly fit a Fitts' Law model $(p<0.001)$.

Figure 6 compares head orientation and EMG control in our SCI participant across the previously described 
(a)

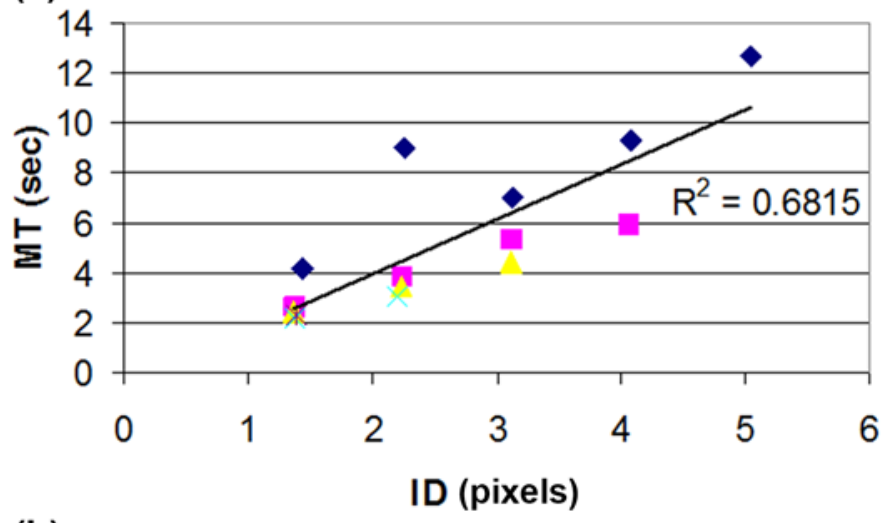

(b)

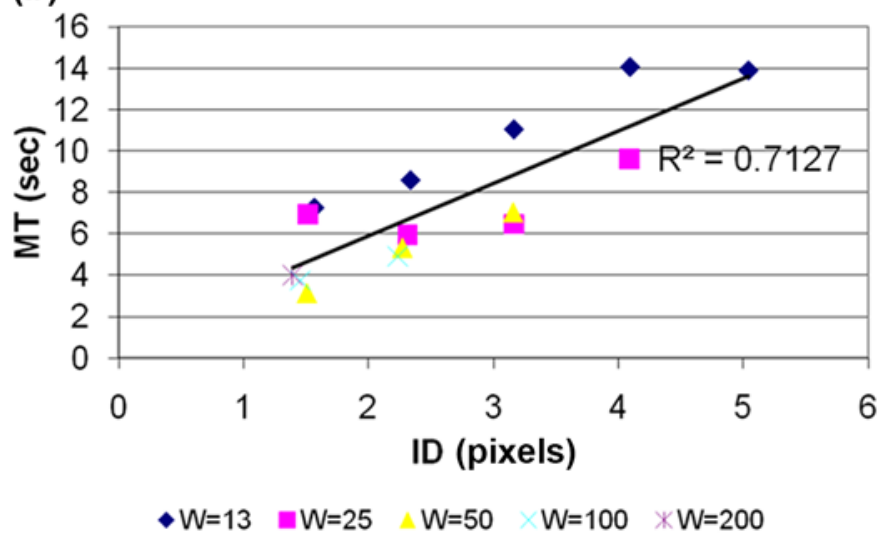

Figure 5.

Regression plots of cursor movement time (MT) to Index of Difficulty (ID). (a) Head orientation-commanded cursor motion.

(b) Electromyography-commanded cursor motion.

performance metrics and also with the performance of these command sources from nondisabled subjects (previously published data). For the SCI subject studied here, there were significant differences between command sources across all performance measures $(p<0.004$, Table 2). Head orientation exhibited superior performance in throughput, path efficiency, and the amount of overshoot compared with EMG, but had lesser performance in terms of reaction time and average speed (Figure 6). Compared with previously published nondisabled performance [24], no statistical difference was observed for our SCI subject for head orientation or EMG in terms of throughput ( $p=0.06$ and 0.09 , respectively) or path efficiency ( $p=0.44$ and $p=0.47$, respectively) (Figure 6). The SCI participant had significantly less overshoot than nondisabled subjects while using head orientation $(p<0.001)$ but no significant difference while using

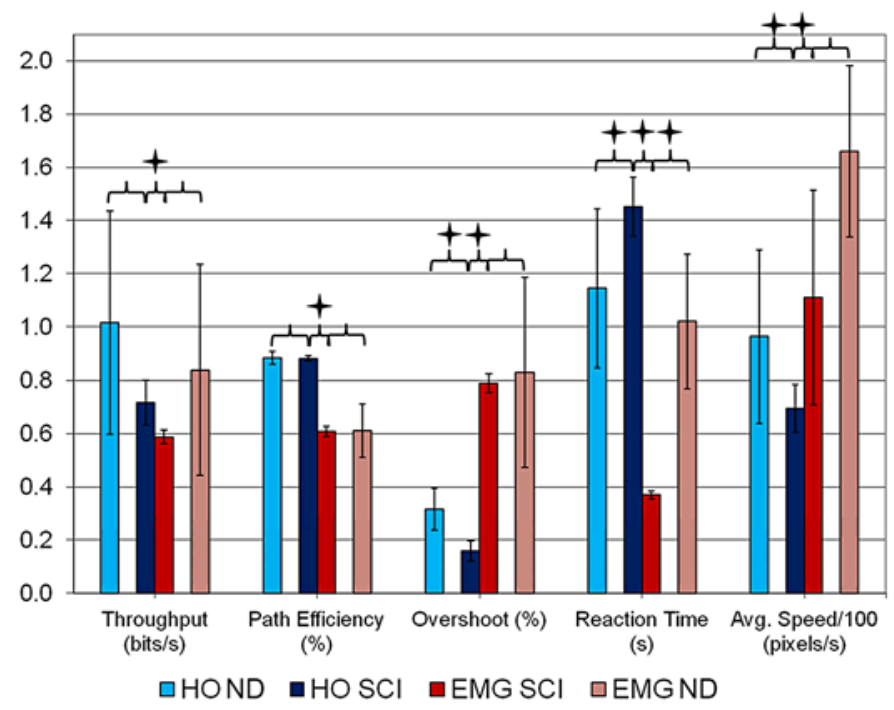

Figure 6.

Command source performance measures compared across sources. Pairwise comparisons are shown with brackets. Comparisons that are significantly different $(p<0.05)$ within marked groups are indicated with star. Avg. = average, EMG = electromyography, $\mathrm{HO}=$ head orientation, $\mathrm{ND}=$ nondisabled, $\mathrm{SCl}=$ spinal cord injury.

EMG $(p=0.40)$. Similarly, the average speed of the SCI participant using head orientation was significantly slower $(p=0.03)$ than nondisabled performance, though for EMG, it was not $(p=0.11)$. The SCI subject's head orientation reaction time was significantly greater than nondisabled performance $(p=0.02)$, while the EMG reaction time was significantly shorter $(p<0.001)$.

The effect of movement direction on performance was quantified by the computed direction ratios, which are the ratio of average performance when the cursor task was diagonal in nature (i.e., required both $x$ and $y$ movements) to the average performance when the cursor task required only an $x$ or only a $y$ command. Overall direction ratios for the SCI participant as well as their comparison to previously published nondisabled data are shown in Table 3 for both command sources. For the SCI user, between command sources, only reaction time showed a significant difference in direction ratio $(p=0.03)$ (Table 3). For head orientation, when compared with nondisabled subjects, the SCI participant showed a significant difference in the direction ratio for path efficiency ( $p=0.03$ ) (Table 3). All other direction ratios were not significantly different from nondisabled ( $p>0.21)$. While using the EMG command source, the SCI participant had throughput and overshoot 
Table 2.

Summary of command source performance by participant with spinal cord injury. Both head orientation (HO) and electromyography (EMG) command sources were significantly different $(p<0.05)$ across all performance measures.

\begin{tabular}{lccc}
\hline $\begin{array}{c}\text { Performance } \\
\text { Measure }\end{array}$ & HO & EMG & p-Value \\
\hline $\begin{array}{c}\text { Throughput } \\
\text { (bits/s) }\end{array}$ & $0.72 \pm 0.12$ & $0.57 \pm 0.14$ & 0.004 \\
$\begin{array}{c}\text { Path Efficiency } \\
\text { (\%) }\end{array}$ & $88 \pm 4$ & $61 \pm 12$ & $<0.001$ \\
$\begin{array}{c}\text { Overshoot } \\
\text { (\%) }\end{array}$ & $16 \pm 22$ & $83 \pm 67$ & 0.002 \\
$\begin{array}{c}\text { Reaction Time } \\
(\text { s) }\end{array}$ & $1.45 \pm 0.52$ & $0.37 \pm 0.05$ & $<0.001$ \\
$\begin{array}{c}\text { Average Speed } \\
\text { (pixels/s) }\end{array}$ & $70.00 \pm 49.19$ & $135.00 \pm 52.25$ & 0.001 \\
\hline \hline
\end{tabular}

direction ratios that were significantly different from nondisabled performance ( $p=0.009$ and $p<0.001$, respectively) (Table 3). No other direction ratios for this command source were significantly different between user populations $(p>0.10)$.

\section{DISCUSSION}

In this work, we studied the performance of two human-computer interfaces-head orientation and face and neck muscle EMG signals - that could be used by an individual with a high cervical SCI to control a twodimensional computer cursor or (potentially) other external actions. The performance of these command sources by the SCI subject were compared with one another and also with previously published nondisabled performance with the same interfaces [24].

\section{Properties of Head Orientation and EMG Command Sources for Subject with SCI}

In general, head orientation appeared to be the more accurate, though somewhat slower, user interface for our SCI subject. Head orientation exhibited a higher overall throughput and path efficiency and lower overshoot, though with slower reaction time and average speed (Figure 6). Both command sources exhibited a high amount of directionality, specifically exhibiting poorer performance for diagonal targets relative to horizontal or vertical targets (Table 3), but this directionality was essentially identical to that described previously for nondisabled subjects for most performance measures. The reduced performance in directional cursor movements reflects the subject's use of several sequential commands (moving first in one direction, then the other) rather than a single diagonal command with both head orientation and EMG interfaces (Figures 3-4). While using head orientation, the subject showed similar patterns of sequential commands for movements to the upper-left and lower-right targets, first moving in one direction and then the other (e.g., upward then to the left). Movements to the other diagonal targets were not as consistent but still showed a mix of both sequential and simultaneous movements. The reason for this preference of movement sequence order is unknown. Both command sources followed a Fitts' Law model of performance, though the smallest targets deviate slightly from this model (Figure 5).

\section{Comparing SCI and Nondisabled Performance}

Overall, the SCI subject's performance with each command source was similar to that reported for a nondisabled population using the same interfaces and performing the

Table 3.

Direction ratios for participant with spinal cord injury (SCI) and previously published nondisabled data (denoted by asterisk). Bolded numbers represent significantly different comparisons $(p<0.05)$ between subject populations and between command sources for participant with SCI.

\begin{tabular}{|c|c|c|c|c|c|c|c|}
\hline Performance Measure & \multicolumn{3}{|c|}{ HO } & \multicolumn{3}{|c|}{ EMG } & $\begin{array}{c}\text { SCI HO } \\
\text { to EMG } \\
\text { (p-Value) }\end{array}$ \\
\hline Throughput (bits/s) & $0.55 \pm 0.10$ & $0.60 \pm 0.05$ & 0.21 & $0.59 \pm 0.20$ & $0.34 \pm 0.04$ & 0.009 & 0.62 \\
\hline Path Efficiency (\%) & $0.79 \pm 0.05$ & $0.83 \pm 0.01$ & 0.03 & $0.72 \pm 0.16$ & $0.61 \pm 0.03$ & 0.10 & 0.33 \\
\hline Overshoot (\%) & $2.17 \pm 2.04$ & $1.87 \pm 0.38$ & 0.70 & $1.35 \pm 0.17$ & $0.96 \pm 0.09$ & $<0.001$ & 0.30 \\
\hline Reaction Time (s) & $1.13 \pm 0.18$ & $1.09 \pm 0.08$ & 0.55 & $0.92 \pm 0.18$ & $0.98 \pm 0.12$ & 0.42 & 0.03 \\
\hline
\end{tabular}


same task [24]. The performance of the implanted EMG interface for the SCI subject was nearly identical to that of the nondisabled population, with the only statistical difference across the various metrics being that the SCI user's reaction time was actually shorter (Figure 6). This shorter reaction time is presumably due to the daily use and practice with the EMG command source by the SCI subject, who used the same approach at home on a daily basis to control a computer cursor. It is interesting to note that while daily practice has perhaps made the SCI subject quicker "off the line," his performance in other metrics is comparable to nondisabled subjects who did not have the benefit of daily practice (Figure 6). The difference in reaction time between populations is not thought to be due to the differences in EMG recording (implanted vs surface) as the large majority of the time to reach movement threshold is due to the cognitive processes of determining which muscles to use for the task and initiating voluntary contraction.

In contrast to the performance of the EMG interface, there were more notable differences between our SCI subject and the nondisabled population for the head orientation interface. Specifically, the overshoot metric was lower for our SCI subject (a desired property), while reaction time and average speed were both significantly slower for the SCI subject than nondisabled control subjects (Figure 6). While head orientation control was statistically slower for the SCI participant, the actual differences were not egregious, and because of the otherwise good performance, this probably would not be a major detriment in a user interface.

\section{Head Orientation Compared with EMG as Command Source}

The SCI subject in this study was able to use both command sources to effectively control a computer cursor with somewhat comparable performance, though this was accomplished using different strategies. Head orientation was a slower but more accurate user interface with less overshoot. The EMG interface produced a very quick motion but tended to overshoot the target, requiring fine adjustments near the final destination to acquire the target. These differences between command sources are a result of both the relative thresholds for movement initiation (which favors EMG in terms of reaction time) and the disparity between the command action and the resulting cursor motion (favoring head orientation as the more intuitive and more similar action-to-movement mapping). While head orientation did outperform EMG in perfor- mance measures (particularly those related to accuracy and velocity control), EMG signals as command inputs can be obtained from an implanted system and currently no implanted system for head orientation measurement exists. An implanted EMG interface, with minimal, or possibly no, externally worn equipment, could potentially afford a very inconspicuous means of humancomputer interaction, decreasing the likelihood of the high abandonment rates seen for many prosthetic systems [29]. Implanted sensors do have some detriments, such as the need for surgery, a slight risk of infection, and the need to be powered within the body. However, the use of implanted neuroprostheses and EMG sensors is growing [30-34], increasing the possibility that an implanted EMG system will be attractive as a computer interface for controlling environmental control units and other assistive systems, such as functional electrical stimulation neuroprostheses and service robots.

\section{Relevant Clinical Populations}

The SCI subject who participated in this case study had an incomplete injury at the cervical 1 to 2 level. The approaches studied here (head orientation and EMG control) are likely to have much broader applicability, however. Individuals with cervical SCI from levels 1 to 4 will have muscles with retained function in both the head and neck, and individuals with cervical 3 to 4 SCI will typically have retained head motion. Other populations that exhibit upper-limb movement impairment severe enough to preclude the use of typical user interfaces such as a mouse or joystick, for example, those with stroke, amyotrophic lateral sclerosis, or bilateral amputation, may also benefit from this approach.

\section{CONCLUSIONS}

This study investigated the performance of an individual with a high cervical SCI using head orientation and EMG (from implanted sensors) command sources to emulate the commands of a computer mouse to control cursor position. Overall, head orientation control was more accurate than EMG control, but the EMG interface was more responsive and faster. The performance for targets located on the diagonal was lower with both head orientation and EMG interfaces relative to single-direction targets, which is very similar to the previously reported performance of nondisabled subjects. This was largely due to the subject giving sequential rather than simultaneous $x$ and $y$ 
commands. Although head orientation performed better in some performance measures, EMG signals can be recorded less obtrusively and more reliably and may be the more practical choice as a user interface.

\section{ACKNOWLEDGMENTS}

\section{Author Contributions:}

Developed the concept: M. R. Williams.

Experimental setup: M. R. Williams.

Conducted the experiments: M. R. Williams.

Analyzed the data: M. R. Williams.

Interpreted the results: M. R. Williams.

Performed statistical analysis: M. R. Williams.

Drafted the manuscript: M. R. Williams.

Obtained funding: R. F. Kirsch.

Assisted in interpreting results: R. F. Kirsch.

Edited the manuscript: R. F. Kirsch.

Read, edited, and approved the manuscript: M. R. Williams, R. F. Kirsch. Financial Disclosures: The authors have declared that no competing interests exist.

Funding/Support: This material was based on work supported by the National Institutes of Health, National Institute of Neurological Disorders and Stroke (grant N01-NS-1-2333); the National Science Foundation, Integrative Graduate Education and Research Traineeship (training grant DGE 9972747); and the National Institutes of Health, Integrated Engineering and Rehabilitation Training Grant (grant T32EB004314).

Institutional Review: The Metro Health Medical Center Institutional Review Board approved this study, and informed consent was obtained from the subject.

Participant Follow-up: The authors do not plan to overtly inform the participant of the publication of this study.

\section{REFERENCES}

1. National Spinal Cord Injury Statistical Center. The 2006 annual statistical report for the model spinal cord injury care systems. Birmingham (AL): National Spinal Cord Injury Statistical Center; 2006.

2. Bureau of Labor Statistics. Computer and internet use at work in 2003. Washington (DC): U.S. Department of Labor; 2005.

3. Digital skills gap: Crunched by the numbers: The digital skills gap in the workforce [Internet]. Boston (MA): Burning Glass Technologies; 2015. Available from: http://burning-glass.com/research/digital-skills-gap/

4. Krueger A, Kruse D. Labor market effects of spinal cord injuries in the dawn of the computer age [Internet]. Cambridge (MA): National Bureau of Economic Research; 1995 Oct [cited 2014 Oct 3]. Available from: http://www.nber.org/papers/w5302

5. Drainoni ML, Houlihan B, Williams S, Vedrani M, Esch D, Lee-Hood E, Weiner C. Patterns of Internet use by persons with spinal cord injuries and relationship to health-related quality of life. Arch Phys Med Rehabil. 2004;85(11):1872-79. [PMID:15520984]

http://dx.doi.org/10.1016/j.apmr.2004.07.350

6. Jacobs R, Hendrickx E, Van Mele I, Edwards K, Verheust M, Spaepen A, van Steenberghe D. Control of a trackball by the chin for communication applications, with and without neck movements. Arch Oral Biol. 1997;42(3):213-18. [PMID:9188991] http://dx.doi.org/10.1016/S0003-9969(96)00117-3

7. Lau C, O’Leary S. Comparison of computer interface devices for persons with severe physical disabilities. Am J Occup Ther. 1993;47(11):1022-30. [PMID:8279497] http://dx.doi.org/10.5014/ajot.47.11.1022

8. Struijk LN. An inductive tongue computer interface for control of computers and assistive devices. IEEE Trans Biomed Eng. 2006;53(12 Pt 2):2594-97. [PMID:17152438] http://dx.doi.org/10.1109/TBME.2006.880871

9. Chen YL, Kuo TS, Chang WH, Lai JS. A novel position sensors-controlled computer mouse for the disabled. Proceedings of the 22nd Annual International Conference of the IEEE Engineering in Medicine and Biology Society; 2000 Jul 23-28; Chicago, IL. p. 2263-66.

10. Anson D, Lawler G, Kissinger A, Timko M, Tuminski J, Drew B. Efficacy of three head-pointing devices for a mouse emulation task. Assist Technol. 2002;14(2):140-50. [PMID:14651252] http://dx.doi.org/10.1080/10400435.2002.10132063

11. Rebman CM Jr, Aiken MW, Cegielski CG. Speech recognition in the human-computer interface. Inf Manage. 2003;40(6): 509-19. http://dx.doi.org/10.1016/S0378-7206(02)00067-8

12. Chang GC, Kang WJ, Luh JJ, Cheng CK, Lai JS, Chen JJ, Kuo TS. Real-time implementation of electromyogram pattern recognition as a control command of man-machine interface. Med Eng Phys. 1996;18(7):529-37. [PMID:8892237] http://dx.doi.org/10.1016/1350-4533(96)00006-9

13. Moon I, Kim K, Ryu J, Mun M. Face direction-based human-computer interface using image observation and EMG signal for the disabled. Proceedings of the 2003 IEEE International Conference on Robotics and Automation; 2003 Sep 14-19; Taipei, Taiwan. p. 1515-20.

14. Rosenberg R. The biofeedback pointer: EMG control of a two dimensional pointer. Proceedings of the 2nd International Symposium on Wearable Computers; 1998 Oct 19-20; Pittsburgh, PA. p. 162-63.

15. Yoshida M, Itou T, Nagata J. Development of EMG controlled mouse cursor. Proceedings of the 24th Annual Conference and the Annual Fall Meeting of the Biomedical Engineering Society; 2002 Oct 23-26; Houston, TX.

16. Huang $\mathrm{CN}$, Chen $\mathrm{CH}$, Chung HY. Application of facial electromyography in computer mouse access for people with disabilities. Disabil Rehabil. 2006;28(4):231-37. [PMID:16467058] http://dx.doi.org/10.1080/09638280500158349 
17. Fitts PM. The information capacity of the human motor system in controlling the amplitude of movement. J Exp Psychol. 1954;47(6):381-91. [PMID:13174710] http://dx.doi.org/10.1037/h0055392

18. Card SK, English WK, Burr BJ. Evaluation of mouse, ratecontrolled isometric joystick, step keys, and text keys for text selection on a CRT. Ergonomics. 1978;21(8):601-13. http://dx.doi.org/10.1080/00140137808931762

19. MacKenzie IS, Kauppinen T, Silfverberg M. Accuracy measures for evaluating computer pointing devices. Proceedings of the SIGCHI Conference on Human Factors in Computing Systems; 2001 Mar 31-Apr 5; Seattle, WA. p. 9-16.

20. Jagacinski RJ, Monk DL. Fitts' Law in two dimensions with hand and head movements. J Mot Behav. 1985;17(1): 77-95. [PMID:15140699] http://dx.doi.org/10.1080/00222895.1985.10735338

21. Radwin RG, Vanderheiden GC, Lin M-L. A method for evaluating head-controlled computer input devices using Fitts' law. Hum Factors. 1990;32(4):423-38. [PMID:2150065]

22. LoPresti EF, Brienza DM, Angelo J. Neck movement patterns and functional performance for computer head controls. Proceedings of the 21st Annual Conference and 1999 Annual Fall Meeting of the Biomedical Engineering Society; 1999 Oct 13-16; Atlanta, GA.

23. LoPresti EF, Brienza DM, Angelo J. Head-operated computer controls: Effect of control method on performance for subjects with and without disability. Interact Comput. 2002;14(4):359-77. http://dx.doi.org/10.1016/S0953-5438(01)00058-3

24. Williams MR, Kirsch RF. Evaluation of head orientation and neck muscle EMG signals as command inputs to a human-computer interface for individuals with high tetraplegia. IEEE Trans Neural Syst Rehabil Eng. 2008;16(5): 485-96. [PMID:18990652] http://dx.doi.org/10.1109/TNSRE.2008.2006216

25. Memberg WD, Polasek KH, Hart RL, Bryden AM, Kilgore KL, Nemunaitis GA, Hoyen HA, Keith MW, Kirsch RF. Implanted neuroprosthesis for restoring arm and hand function in people with high level tetraplegia. Arch Phys Med Rehabil. 2014;95(6):1201-11.e1. [PMID:24561055] http://dx.doi.org/10.1016/j.apmr.2014.01.028

26. Soukoreff RW, MacKenzie IS. Towards a standard for pointing device evaluation, perspectives on 27 years of Fitts' law research in HCI. Int J Hum Comput Stud. 2004;61(6): 751-89. http://dx.doi.org/10.1016/j.ijhcs.2004.09.001

27. Smith BT, Mulcahey MJ, Betz RR. Development of an upper extremity FES system for individuals with C4 tetraplegia. IEEE Trans Rehabil Eng. 1996;4(4):264-70.

\section{[PMID:8973952]}

http://dx.doi.org/10.1109/86.547926

28. Evans DG, Drew R, Blenkhorn P. Controlling mouse pointer position using an infrared head-operated joystick. IEEE Trans Rehabil Eng. 2000;8(1):107-17. [PMID:10779114] http://dx.doi.org/10.1109/86.830955

29. Silcox DH 3rd, Rooks MD, Vogel RR, Fleming LL. Myoelectric prostheses. A long-term follow-up and a study of the use of alternate prostheses. J Bone Joint Surg Am. 1993;75(12):1781-89. [PMID:8258548]

30. Kilgore KL, Hart RL, Montague FW, Bryden AM, Keith MW, Hoyen HA, Sams CJ, Peckham PH. An implanted myoelectrically-controlled neuroprosthesis for upper extremity function in spinal cord injury. Conf Proc IEEE Eng Med Biol Soc. 2006;1:1630-33. [PMID:17946056]

31. Kilgore KL, Hoyen HA, Bryden AM, Hart RL, Keith MW, Peckham PH. An implanted upper-extremity neuroprosthesis using myoelectric control. J Hand Surg Am. 2008; 33(4):539-50. [PMID:18406958]

http://dx.doi.org/10.1016/j.jhsa.2008.01.007

32. Troyk PR, DeMichele GA, Kerns DA, Weir RF. IMES: an implantable myoelectric sensor. Conf Proc IEEE Eng Med Biol Soc. 2007;2007:1730-33. [PMID:18002310]

33. Weir R, Troyk P, Demichele G, Kerns D. Technical details of the implantable myoelectric sensor (IMES) system for multifunction prosthesis control. Conf Proc IEEE Eng Med Biol Soc. 2005;7:7337-40. [PMID:17281975]

34. Weir RF, Troyk PR, DeMichele GA, Kerns DA, Schorsch JF, Maas H. Implantable myoelectric sensors (IMESs) for intramuscular electromyogram recording. IEEE Trans Biomed Eng. 2009;56(1):159-71. [PMID:19224729] http://dx.doi.org/10.1109/TBME.2008.2005942

Submitted for publication January 5, 2015. Accepted in revised form July 31, 2015.

This article and any supplementary material should be cited as follows:

Williams MR, Kirsch RF. Case study: Head orientation and neck electromyography for cursor control in persons with high cervical tetraplegia. J Rehabil Res Dev. 2016; 53(4):519-30.

http://dx.doi.org/10.1682/JRRD.2014.10.0244

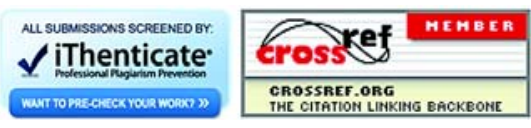

